DOI: https://doi.org/10.14311/TPFM.2017.035

\title{
MULTIFRACTAL ANALYSIS OF A WAKE FOR A SINGLE WIND TURBINE
}

\author{
S. Strijhak ${ }^{1}$, J.M. Redondo ${ }^{2}$, J. Tellez ${ }^{2,3}$ \\ ${ }^{1}$ Institute for System Programming of the Russian Academy of Sciences, Alexander \\ Solzhenitsyn street, 109004 Moscow, Russia \\ ${ }^{2}$ Department of Physics, Technical University of Catalonia, Jordi Girona Street 1-3, \\ 08034, Spain \\ ${ }^{3}$ Institute Flumen, Department of Civil and Environmental Engineering, Technical \\ University of Catalonia, Jordi Girona Street 1-3, 08034, Spain
}

\begin{abstract}
Wind energy is an important component of renewable energy sources. Studying of turbulent wakes for wind turbines and interactions with Atmospheric Boundary Layer is an urgent problem. The theory of chaos and fractal geometry with some research in fluid dynamics will change profoundly the scope of complex scale to scale transport in theory of turbulence, in particular when body forces, such as stratification and rotation are important. For this reason, we present some preliminary ideas and some results of the analysis of the structure function and multi-fractal behaviour of the evolution of turbulence in the wake of a single wind turbine through the scaling analysis using the program ImaCalc. The analysis of fractal dimension was performed on the results of a numerical simulation consisting on a Large-Eddy Simulation and Lagrangian Dynamic Smagorinsky Mode (LDSM)1 for the wind turbine wakes in the Atmospheric Boundary Layer by OpenFoam software. The role of intermittency in wind turbine technology in the stratified Atmosphere is also discussed.
\end{abstract}

Keywords: wind energy, wind turbine, turbulence, wake, experiment, numerical simulation, isotropic turbulent flows, self-similarity dimension, intermittency, large eddy simulation, numerical domain, Lagrangian-averaged scale-independent dynamic Smagorinsky model, Actuator Line Model, velocity, vorticity, nonlinear process, fractal dimension.

\section{Introduction}

Wind energy is an important component of renewable energy sources in connection with the need for electricity energy. Studying of turbulent wakes for wind turbines and interactions of wakes with Atmospheric Boundary Layer (ABL) is an urgent task due to the need of assessment of deficit of velocity in wakes for wind turbines as a part of the wind farm installation [1-4]. Recently intensive researches by means of an experiments [5-8] and numerical modeling [9-14]: Large Eddy Simulation (LES), vortex and analytical models for the purpose of assessment of average and statistical characteristics for turbulence for obtaining the maximum power are conducted. In such flows the wakes, created by large engineering devices, interact with large-scale variable atmospheric flow phenomena.

One of the ways for assessment of statistical behavior of turbulent wakes is based on using structural function which possessing the ability to identify the coherent flow structure and particularly determine isotropy levels through a statistical description. The structure function $B(R)$ of order $p$ is defined in terms of the moments of the velocity increments:

$$
B(R)^{P}=\left(u(x+R)-u(x)^{P}\right)=\delta_{R}(u)^{P}
$$

where $u$ is the streamwise velocity component at location $x, R$ is a spatial separation distance between two points, which indeed quantifies a particular scale of interest.

Kolmogorov (K41) introduced a similarity theory for homogenous and isotropic turbulent flows [15], which states that the mean dissipation energy $\varepsilon$, is scale invariant and the structure function is given by:

$$
B(R)^{P} \sim\langle\varepsilon\rangle^{P / 3} R^{P / 3}
$$

Higher order statistics display discrepancies when comparing the evaluation as proposed by K41 to experimental data is described as intermittency, and this process is taken into account, where measurements show that there is a spatial or temporal variation of the energy dissipation. In most flows, and especially in geophysical flows a significant deviation from the mean is found in the dissipation [16]. 
Kolmogorov (K62) refined the previous K41 similarity theory and presented a log-normal distribution for scale dependence within the dissipation range as [17]:

$$
B(R)^{P} \sim\left\langle\varepsilon_{R}\right\rangle^{P / 3} R^{P / 3} \sim R^{\xi_{p}}
$$

The intermittency phenomenon can be recognized via the scaling exponent of appropriate moments, which associates the separation scales between two neighboring points [18].

$$
\xi_{p}=\frac{p}{3}+\frac{1}{18} \mu p(3-p)
$$

where $\mu$ - is the intermittency exponent, which characterizes the time variation of the fluctuation of energy dissipation. $\xi_{\mathrm{p}}$ is the scaling exponent of order $p$.

Frisch et al. introduced the Beta-model, which is dependent on the energy cascade and concentrates on the transmission of nonlinearity in the inertial subrange [19]. The scaling exponent of the Beta model can be expressed as:

$$
\xi_{p}=\frac{p}{3}+\frac{1}{3}(3-H)(3-p)
$$

where $H$ - is the Hausdroff dimension, otherwise known as the self-similarity dimension of the dissipative structures and in a three dimensional space defined as $H=3-\mu$

The Intermittency in turbulence has been investigated for different types of flows such Atmospheric Boundary Layer flow [20], wake flow for fractal grid, free jet, cylinder [21], wake flow in experiment for $3 \times 3$ wind turbine array [22].

The goal of this work is to define values for $H$ and $\mu$ for the flow with a wake behind a single horizontal-axis wind turbine with 3 blades.

\section{Mathematical model and problem definition}

The mathematical model included the main equations of mass and momentum conservation laws for incompressible flow. The large-scale vortex structures paid off by means of integration the filtered equations [14,23]. For receiving the filtered equations the box filter was used. The small eddies, for which the size didn't exceed a size of grid cell, were modelled by means of a Lagrangian-averaged scaleindependent (LASI) dynamic Smagorinsky model [24].

$$
\frac{\partial \bar{u}_{j}}{\partial x_{j}}=0
$$

where $\bar{u}_{j}=u_{j}-u_{j}^{\prime}$ - vector of value for resolved-scale velocity after the procedure of a filtration the equations.

$$
\frac{\partial \bar{u}_{i}}{\partial t}+\frac{\partial}{\partial x_{j}}\left(\bar{u}_{j} \bar{u}_{i}\right)=-2 \varepsilon_{i j k} \Omega_{j} \bar{u}_{k}-\frac{\partial \widetilde{p}}{\partial x_{i}}-\frac{\partial}{\partial x_{j}}\left(R_{i j}^{D}\right)+\left(\frac{\rho_{b}}{\rho_{0}}-1\right) g_{i}-\frac{\partial p}{\partial x_{i}}+f_{i}
$$

where $\varepsilon_{\mathrm{ijk}}$ - is the alternating unit tensor, $\Omega_{\mathrm{j} \text { - }}$ is the planetary rotation rate vector at the point of interest on the planet, $\tilde{p}$ - is pressure, $R_{i j}^{D}$ - is the deviatoric part of the sub-grid-scale (SGS) stress tensor. $\rho_{\mathrm{b}} / \rho_{0}$ the term which takes into account the buoyancy force, $g_{\mathrm{i}}$ - is the gravitation vector, $\partial p / \partial x_{i}$ - the gradient for pressure in horizontal direction.

The mathematical model was realized in SOWFA (Simulator for On/Offshore Wind Farm Application) code. SOWFA is an OpenFOAM-based incompressible atmospheric/wind farm Large-Eddy Simulation (LES) solver, which is based on finite-volume method, which models turbines as actuator lines and actuator disk [11-12].

The numerical domain was set from point $\left(\begin{array}{lll}0 & 0 & 0\end{array}\right)$ as: $\left(\begin{array}{lll}-378 & -378 & -378\end{array}\right)$ meters to the left side, and (2646 378 378) to the right side of numerical domain. The location of horizontal-axil NREL 5 MW wind turbine was defined in the point with coordinates: (5.0 0.0 -90.0) meters.

The number of blades for wind turbine was 3, the radius of blade was 63.0 meters, the radius of turbine's hub was 1.5 meters, the tower height was 87.6 meters, the rotor speed was $9.15 \mathrm{rpm}$.

The background mesh was built with blockMesh utility, the refined mesh with refineMesh utility. The refineMesh is used to refine a region around turbine and in wake. 
The size of cell for background mesh was $\Delta x=\Delta y=\Delta z=31$ meters. The total number of hexahedral cells after refinement procedure for mesh was 2979 840. At least 40 cells were set across diameter of rotor for resolving tip and root vortices.

The value of wind velocity at inlet boundary for numerical domain $U_{\mathrm{G}}=(8.0,0.0,0.0) \mathrm{m} / \mathrm{s}$ was set. At the top and bottom boundaries, slip flow condition was set for value of velocity, on side faces cyclic conditions were set, and at output boundary the condition of continuation for solution was set. For pressure at side boundary, the cyclic condition was set; on other boundaries the equality condition to zero gradient was set. The numerical domain and the adapted unstructured grid are shown in Figure 1.

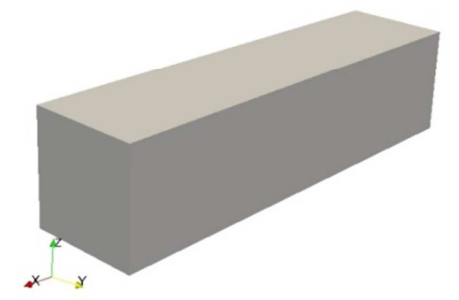

a)

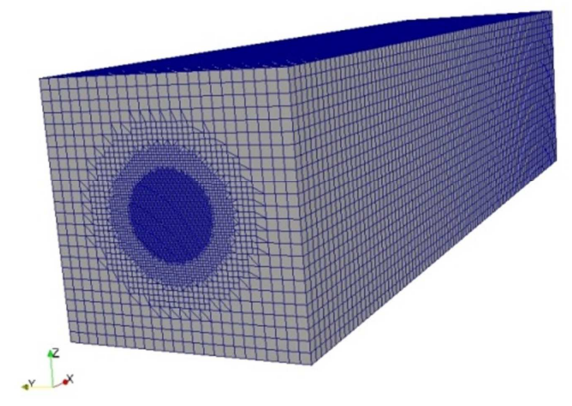

b)

Figure 1: a) numerical domain, b) numerical grid

Considering the characteristic sizes of blades of wind turbine, Re numbers can reach an order of $\mathrm{Re}=10 \mathrm{e} 7-10 \mathrm{e} 8$, it is difficult to resolve all scales of flow by means LES. For this purpose too big numerical grids will be required. It is known that approach on the basis of Actuator Line Model (ALM) doesn't demand too detailed grids around the turbine blades. This approach allows reproducing various types of vortices: in a wake, trailer, root and boundaries. In the scope of ALM the blade of the turbine is sampled into separate flat sections with the set geometry, in the form of the known profiles with the set thickness and a twist of a profile. For each profile values of lift and drag forces are calculated. The force operating on a flow is equal on the module to force of the turbine operating on the blade. Further the procedure of rationing of force is carried out and the size of force is projected on a flow by addition separate term in the momentum equation for the number. The resultant force of $\mathrm{F}$ was determined by a technique [25]:

$$
f_{i}^{\text {turbine }}(r)=\frac{F_{i}^{\text {actuator }}}{\varepsilon p s^{3} \pi^{3 / 2}} \exp \left[-\left(\frac{r}{\varepsilon p s}\right)^{2}\right]
$$

where $F_{\mathrm{i}}^{\text {actuator }}$ is the actuator point force, $f_{\mathrm{i}}^{\text {turbine }}$ is the force projected as a body force onto Computational Fluid Dynamics (CFD) grid, $r$ - is the distance between CFD cell center and actuator point, eps controls width of Gaussian distribution.

Data for coefficients of drag force $C_{\mathrm{x}}(a)$, lift force $C_{\mathrm{y}}(a)$ with changing of angle of attack (AoA) $a$ from $-180^{\circ}$ till $180^{\circ}$ for simple bodies, which describe a wind turbine: "Cylinder1, "Cylinder2", list of airfoils used to define blade: "DU40_A17" (DU-W-405LM), "DU35_A17" (DU-W-350), "DU30_A17" (DU 97-W-300LM), "DU25_A17" (DU 91-W2-250LM), "DU21_A17" (DU 93-W-210LM), "NACA64_A17" (NACA 64-618) are defined in special table. The step for AoA was defined as $5^{\circ}$. The values of thickness for profile and its twist were also set. The Figure 2 shows the main scheme for Actuator Line Model with actuator elements. 

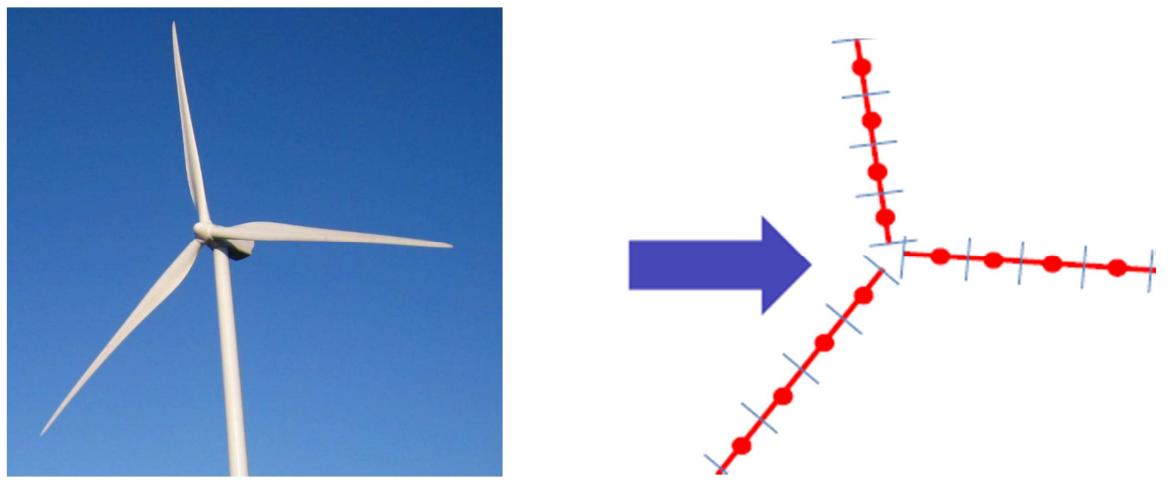

Figure 2: Photo of wind turbine with 3 blades and scheme for Actuator Line Model

The number of actuator elements per blade of wind turbine was set equal to 64. The distribution of provision of sections on length for the blade was selected by uniform. The value of the epsilon parameter eps (width of Gaussian distribution) in a Formula 8 was set equal 10.0.

All physical values in numerical domain were defined at the center of a numerical cell [26]. Approximation of terms in equations was executed with the second order of accuracy on time and space. The equations for coupling of velocity, pressure, were solved by means of an iterative algorithm PIMPLE. All methods (ALM, PIMPLE, special boundary conditions for ABL) were realized in pisoFoamturbine solver. The linear system of algebraic equations was solved by iterative method of conjugate gradients with a preconditioner for velocity, pressure, a stress tensor and parameters for model of subgrid viscosity. A total quantity of the calculated physical values (scalar, vector, tensor) for the selected model for subgrid-scale turbulent viscosity was equal to 18 . In this regard the resources of highperformance cluster were required. The value of stress tension of the surface can be determined by the Schumann model. On a surface the components of stress tensor are equal to zero, except values $\tau 13, \tau 23$.

\section{Results of LES simulation}

During the calculation fields of average (mean) and instant values (velocity, pressure, subrid viscosity and energy, a stress tensor and others) have been received. The general time of simulation was 1000 seconds, the time step was set by $\mathrm{dt}=0.033$ seconds. The maximum Courant number Co was equal to 0.11 . In the Figure 3 the results of calculation for value of velocity magnitude for different time are presented.

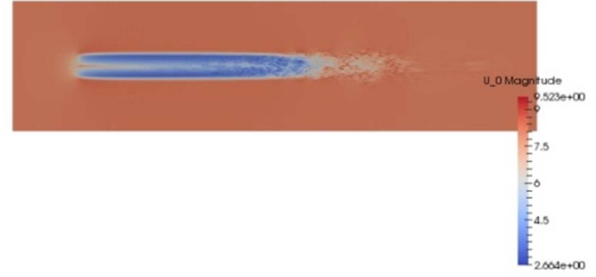

a)

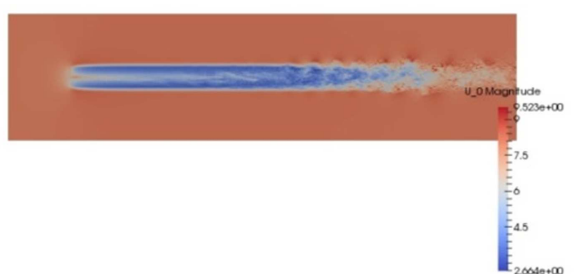

c)

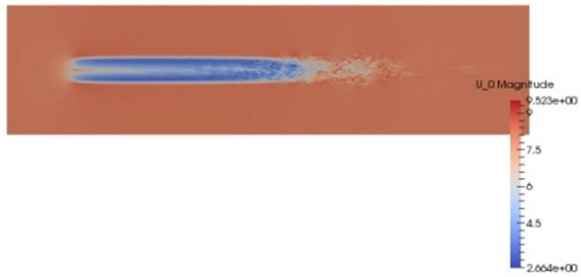

b)

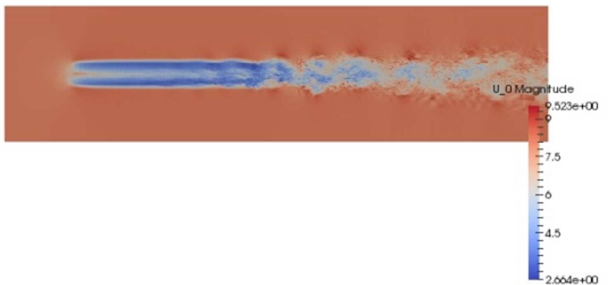

d)

Figure 3: U_0 magnitude value at different time a) $150 \mathrm{~s}$, b) $300 \mathrm{~s}$, c) $500 \mathrm{~s}$, d) $1000 \mathrm{~s}$

The vorticity $(\omega=\nabla \times U)$ and Reynolds stress are shown in Figures 4 and 5 .

It is visible that the turbulent wake for wind turbine changes in time and extends to output boundary of numerical domain. The first results of calculations show that final time in 1000 seconds is sufficient for the first assessment of a picture of an unsteady flow. A large number of temporary steps demands large computing resources. In the Figure 6, dependencies of distribution for total and average pressure, velocity 
on quantity iterative steps on time are presented. A record time's step is equal to $\mathrm{d} t=0.033$ second. It is visible what in the location of wind turbine the value for pressure sharply changes.

In the Figure 7 dependences for kinetic energy of turbulence and turbulent viscosity on the number of iterations on time are presented. Their distributions have close character. In the Figure 8 dependences for average values of nonlinear sizes for a fluctuation of velocity components from the number of iterations on time are presented. These dependences characterize anisotropy of the flow. The greatest values have sizes on with components of axes OZ and OZ that is characteristic of a flow with rotation of flow.

In the Figure 9, dependences for average sizes stress tensor component from the number of iterations on time are presented. Distribution a component on axes OX and OX, OY and OY has close character and gives the maximum contribution to the general of value of the module of stress tensor.

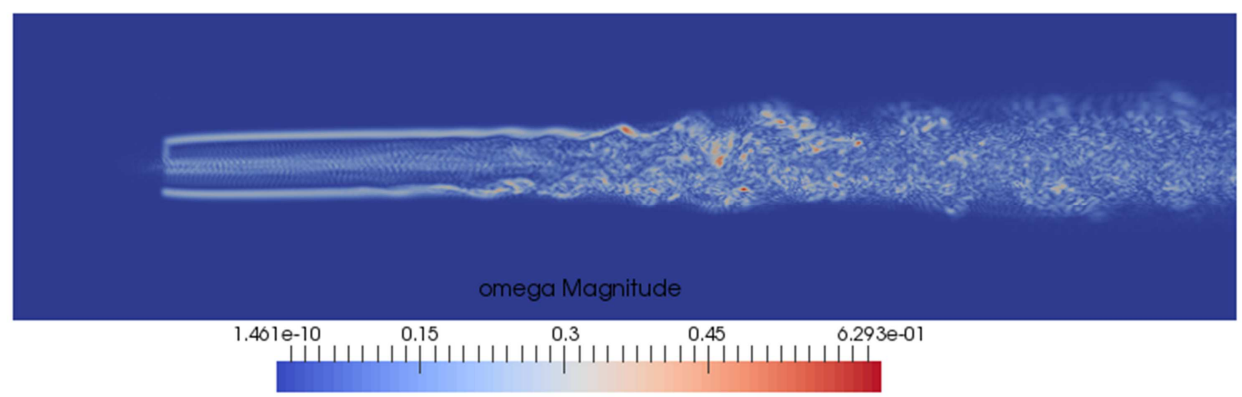

Figure 4: Vorticity (omegaMagnitude) at $\mathrm{t}=1000 \mathrm{~s}$

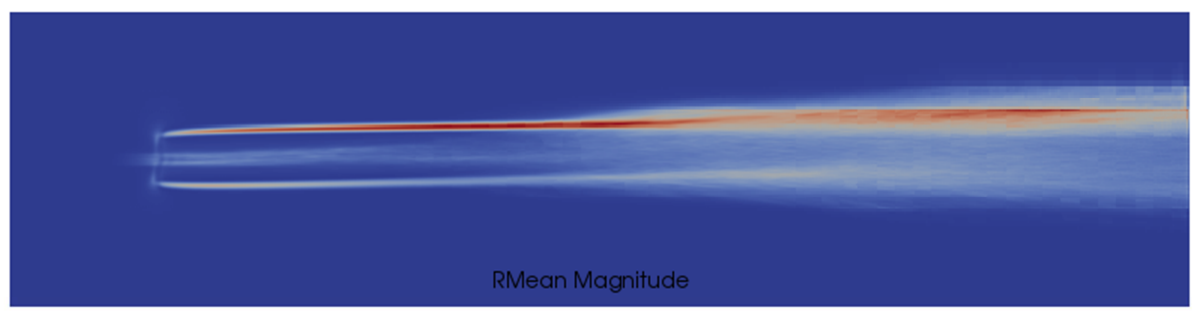

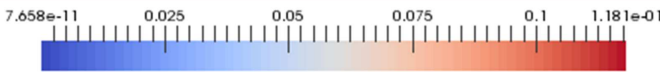

Figure 5: Reynolds stress at $\mathrm{t}=1000 \mathrm{~s}$

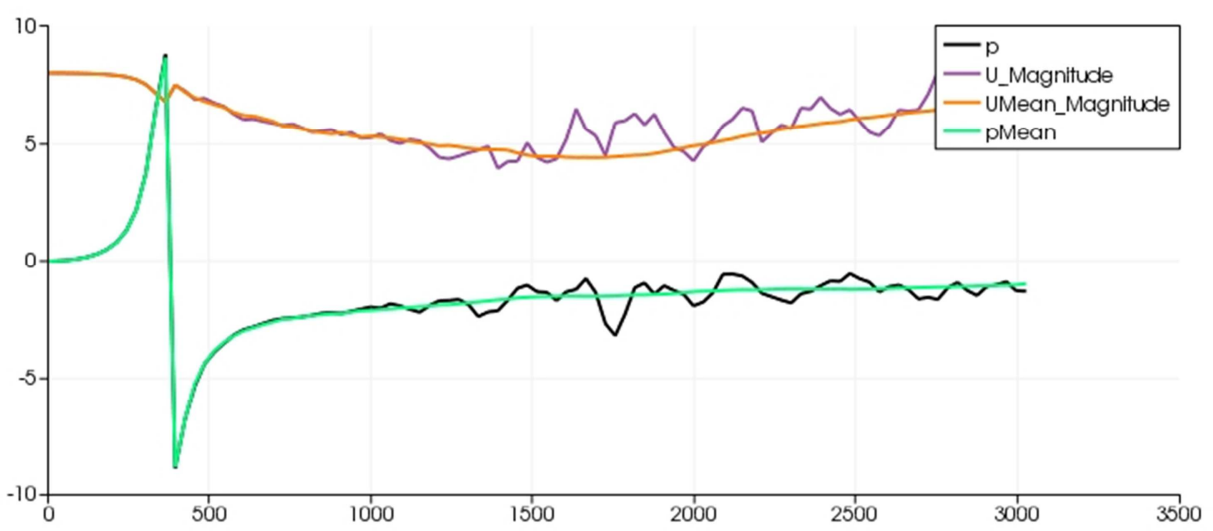

Figure 6: Total and Mean values of pressure, velocity from number of iterations 


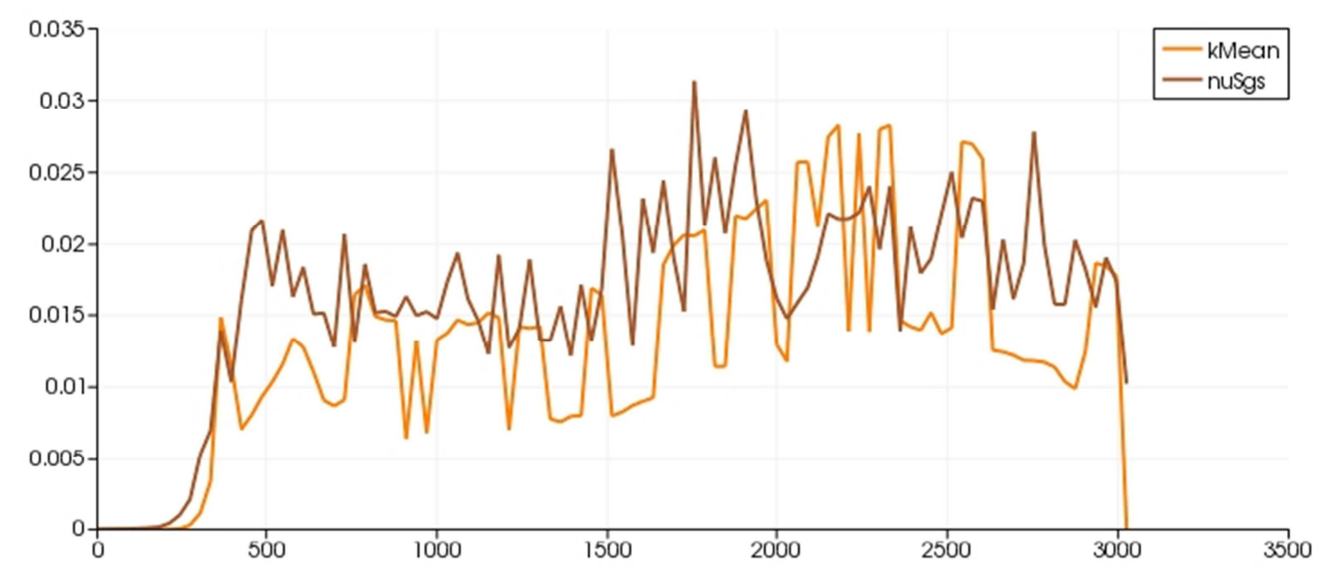

Figure 7: Subgrid scale turbulent kinetic energy and viscosity from number of iterations

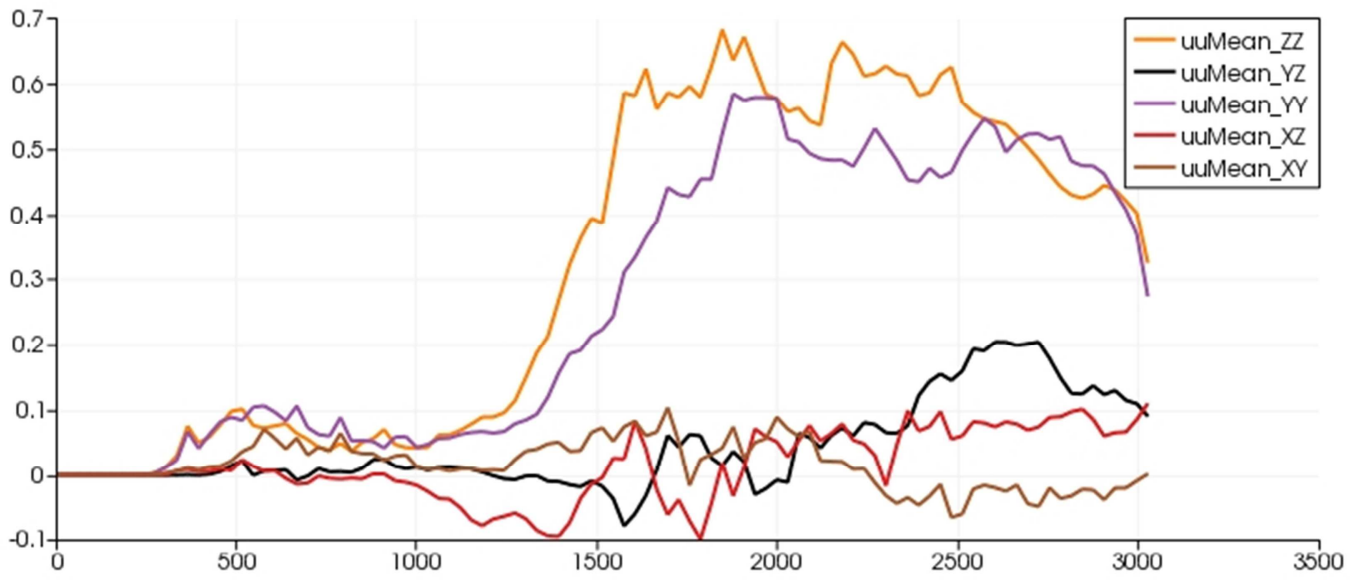

Figure 8: Subgrid scale mean values of nonlinear fluctuation components of velocity from number of iterations

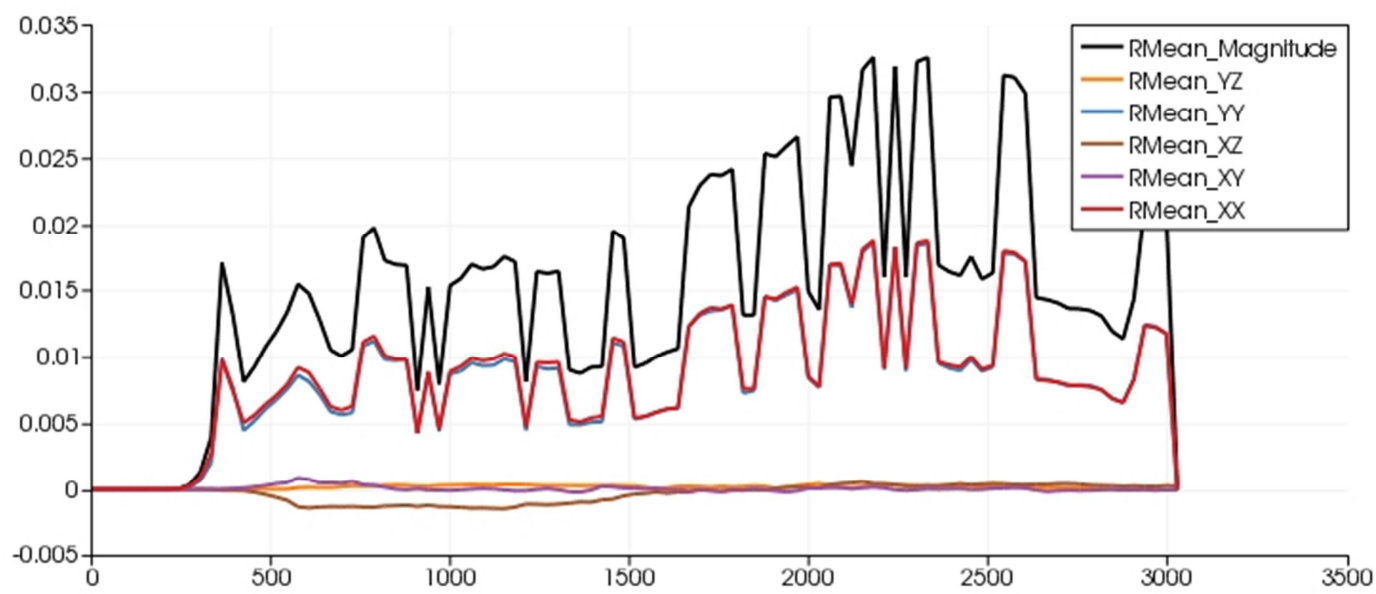

Figure 9: Subgrid scale mean values of stress tensor components from number of iterations

\section{Calculation of Fractal Dimensions}

Fractal objects are irregular in shape but their irregularity is similar across many scales, enabling them to be described mathematically and to be generated computationally. We apply these advances of fluid visualization to investigate different scale to scale properties of the complex wind turbine wakes through 
simple iterative box-counting algorithms for the different values of velocity and vorticity. These techniques are similar to spectral methods and take advantage of programs described in [27] (Digiflow, Imacal, Matlab). Here we consider multifractals as a statistic distribution that yields useful information even if the underlying structure does not show a complete self-similar or self-affine behavior, we consider the vorticity modulus from the LES Open Foam simulations as the relevant flow descriptor.

The measurement of multifractals is mainly the measurement of a statistic distribution which is why the results yield useful information even if the underlying structure does not show a self-similar or self-affine behavior. For a monofractal object, the number $n$ of features of a certain size $\delta$ varies as

$$
n(\delta) \propto \delta^{-D_{0}},
$$

where the fractal dimension $\mathrm{D}_{0}$

$$
D_{0}=\lim _{\delta \rightarrow 0} \frac{\log n(\delta)}{\log \frac{1}{\delta}}
$$

can be measured by counting the number $n$ of boxes needed to cover the object under investigation for increasing box sizes $\delta$ and estimating the slope of a log-log plot.

There are several methods for implementing multifractal analysis; in this section the moment method (27, 28 ) is explained. This method uses mainly three functions: $\tau(q)$, mass exponent function, $\alpha$, coarse Hölder exponent, and $f(\alpha)$, multifractal spectrum. For a measure (or field) defined in a two-dimensional support of the $L x L$ pixels image, $\mu$ (grey tone from 0 to 255 ), it could be spatially decomposed in terms of infinitely many intertwined sets of fractal dimensions. If that is the case, one fractal dimension cannot characterize all the complexity and several fractal dimensions will be estimated depending on the position.

Applying box counting "up-scaling" partitioning process we can get the partition function $\chi(q, \delta)$ defined as :

$$
\chi(q, \delta)=\sum_{i=1}^{n(\delta)} \mu_{i}^{q}(\delta)=\sum_{i=1}^{n(\delta)} m_{i}^{q}
$$

Where $m$ is the mass of the measure, $q$ is the mass exponent, $\delta$ is the length size of the box and $n(\delta)$ is the number of boxes in which $m_{i}>0$. Based on this, the mass exponent function $\tau(q)$ shows how the moments of the measure scales with the box size:

$$
<\tau(q)>=\lim _{\delta \rightarrow 0} \frac{\log <\chi(q, \delta)>}{\log (\delta)}=\lim _{\delta \rightarrow 0} \frac{\log <\sum_{i=1}^{n(\delta)} m_{i}^{q}>}{\log (\delta)}
$$

where $<>$ represents statistical moment of the measure $\mu_{i}(\delta)$ defined on a group of non-overlapping boxes of the same size partitioning the area studied. $D_{\mathrm{q}}$ are related as $\tau(q)=(1-q) D_{q}$.

This characterization of multifractal measures is the concept of generalized dimensions $D_{\mathrm{q}}$, which corresponds to the scaling exponents for the $\mathrm{q}^{\text {th }}$ moment of the measure, defined as

$$
D_{q}=\lim _{\delta \rightarrow 0} \frac{1}{1-q} \frac{\log \sum_{i=1}^{n(\delta)} m_{i}^{q}}{\log \delta}
$$

The sum in the numerator of Equation 12 is dominated by the highest values of $\mathrm{p}_{\mathrm{i}}$ for $\mathrm{q}>0$, and by the lowest values of $\mathrm{p}_{\mathrm{i}}$ for $\mathrm{q}<0$. The singularity index $(\alpha)$ can be determined by Legendre transformation of the $\tau(q)$ curve as:

$$
<\alpha(q)>=\frac{d<\tau(q)>}{d q}
$$

The number of cells of size $\delta$ with the same $\alpha, n_{\alpha}(\delta)$, is related to the cell size as $n_{\alpha}(\delta) \propto \delta^{-f(\alpha)}$, where $f(\alpha)$ is a scaling exponent of the cells with common $\alpha$. Parameter $f(\alpha)$ can be calculated as:

$$
<f(\alpha)>=q<\alpha(q)>-<\tau(q)>
$$

Multifractal spectrum (MFS), a graph of $\alpha$ vs. $f(\alpha)$, quantitatively characterizes variability of the measure studied with asymmetry to the right and left indicating domination of small and large values 
respectively. The width of the MF spectrum indicates overall variability and all are factors which characterize the type and scaling properties of the turbulence.

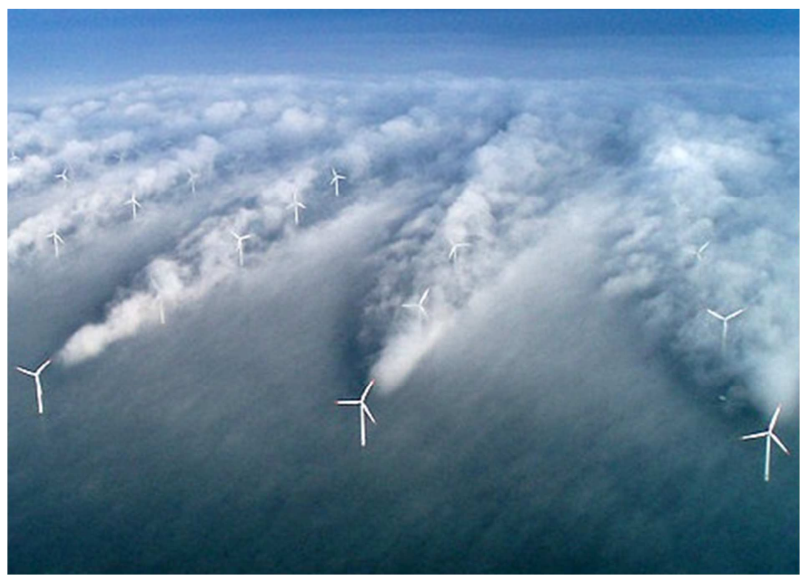

Figure 10: Array of wind mills in the ABL, with their wakes marked by condensation of water vapour

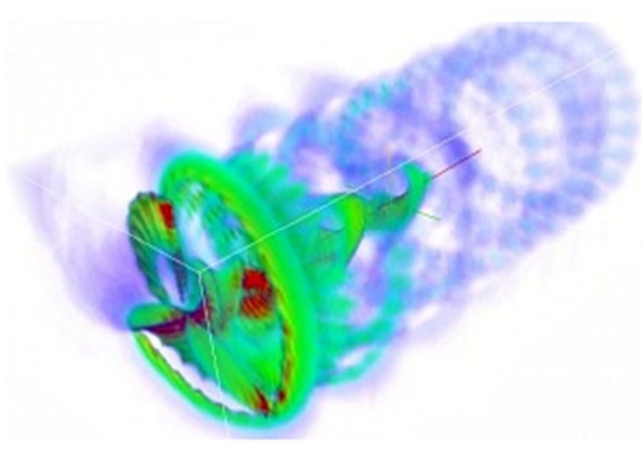

Figure 11: The structure of the close up wake of a rotating wind mill

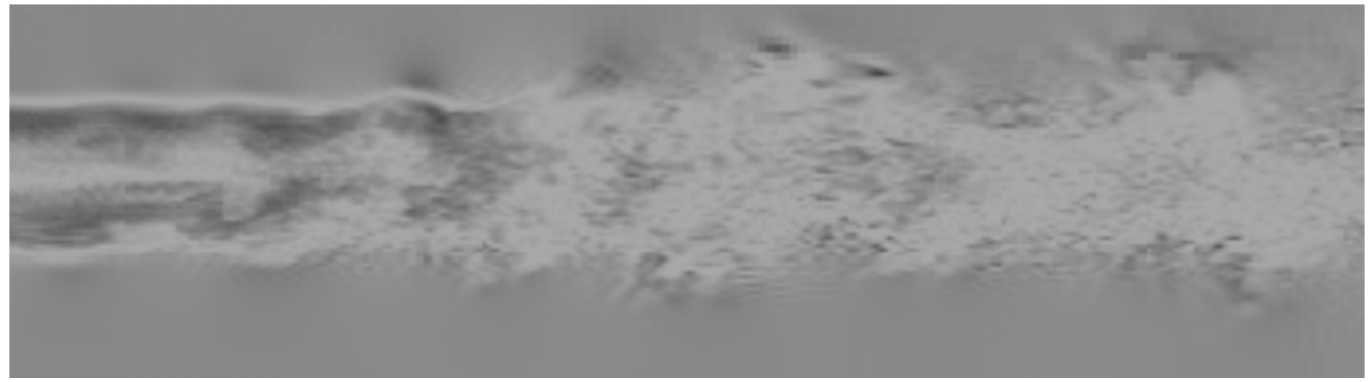

Figure 12: Detail of the vorticity wake at $\mathrm{t}=600$ seconds

\section{Results and Discussion}

Imacal program is used to study nonlinear process and fractal parameter. Here we calculate the maximum value of fractal dimension selected among all of the velocity intensity and of the vorticity modulus shown in figure 4 which is a good indicator of the complexity of the flow. The data of velocity magnitude fields U_0 from $\mathrm{t}=150 \mathrm{sec}$ till $\mathrm{t}=1000 \mathrm{~s}$ and the analysis at different sections of the numerical domain (in the middle along $\mathrm{OX}$ axis, and on the tip blade"s section) have also been used. The data of calculation and the evolution of $\mathrm{D}(\mathrm{t})$ at different times in the middle section of numerical domain along main axis are also calculated. On Figures 10 and 11 show the structure of the wakes. The similar methodology in the velocity field carried out in the centerline and in the section on the end of the blade has shown small differences in the maximum fractal spectra. [29, 30]. The typical shape of an equilibrium turbulence spectra is parabolic [28].

In Figure 12 a close up description of the central plane of vorticity during the time corresponding to 600 seconds, in this preliminary study only selected times and positions have been measured, but some conclusions may be inferred from the topology of the vorticity iso-contours.

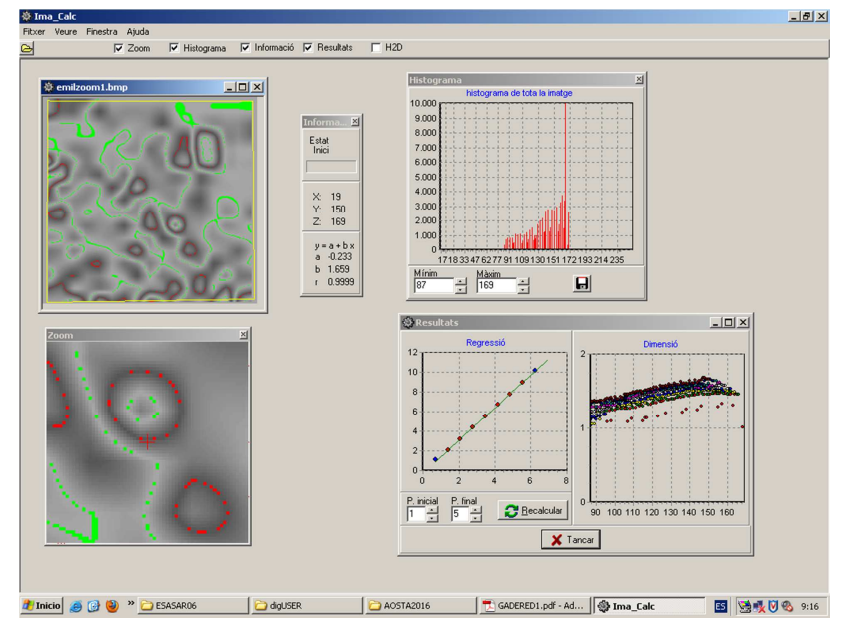

Figure 12: A multiple analysis of the most convoluted vorticity contour of the Wind mill wake at $t=600 \mathrm{~s}$ 


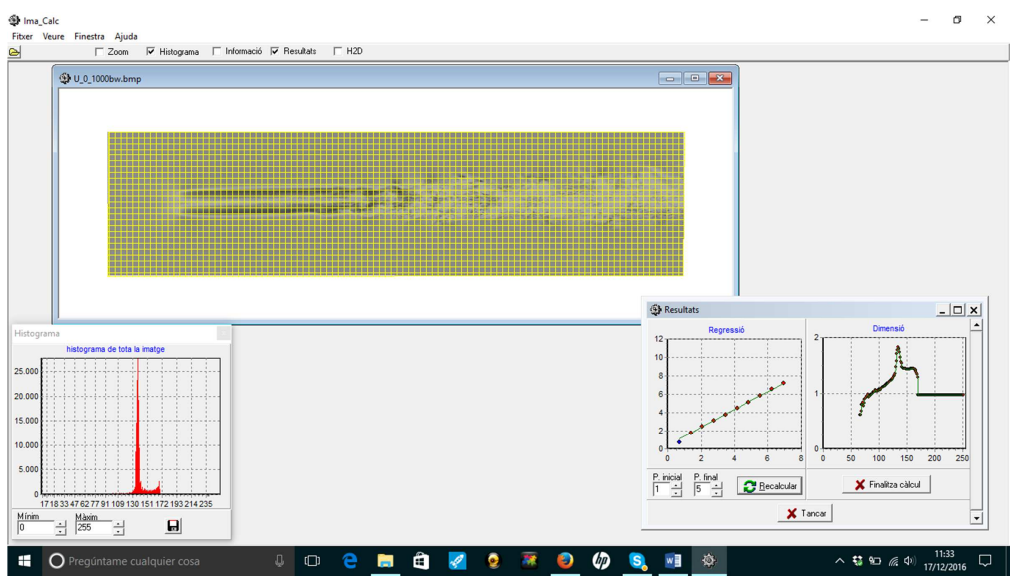

Figure 14: An example of ImaCalc output for a vorticity wake at $\mathrm{t}=1000$ seconds, showing the vorticity histogram and a limited range of fractal dimension between intensities of 60 and 160 . The maximum fractal dimension, for 138 ; corresponds to value of about 1.90 .

\section{Conclusions}

The structure of the wakes of the wind turbines, show their complexity combining the effects of rotation and stratification due to the initial logarithmic wind profiles used. The results qualitatively match the known data of similar large scale experiment and numerical calculations, The observations that vorticity contours are smoother than the velocity ones, provide an opportunity to adequately calculate also the scaling of other important flow parameters (velocity, pressure, vorticity, shear, stress tensor) for a wake of single wind turbine. The ImaCalc program was used to calculate the value of fractal dimension for the vorticity contours. The intermittency exponent changed for higher values of vorticity at an increasing rate, from 1.1 for low values up to the maximum of 2.1. The corresponding values of $H$ varied from 0.9 to 1.9. So the intermittency exponent $\mu$, according to equation (5), changes with the levels of vorticity values for a developed vorticity wake behind a wind turbine.

The calculations were run using resources of a high-performance cluster with 12-96 cores for each single case, in the scope of UniHUB web-laboratory of ISPRAS.

It is planned further to perform researches of nonlinear dynamics of vortex structures in the wakes of more complex and higher Reynolds flows. (Energy spectrum of velocity, Probability density function of velocity increments. The scaling exponents and velocity PDF's have been studied by [20,27] Showing that the third order structure scaling exponent is not exactly 3 , so Intermittency acts as a natural compensating effect to the scale to scale transfer of energy (and enstrophy) in non-homogeneous flows [30,31]. The high order statistics of velocity increments: skewness and flatness for hub height and tip regions of wind turbine) are key examples of how to improve simulations taking into account different subgrid-scale models for LES, and comparing higher order influences of changes of value of the heat flux at the Earth's surface, modifying buoyancy, or on the dynamics of rotation of the turbine and its influences on the turbulent wake and its intermittency.

\section{References}

[1] Vermeer, L., Sørensen, J. N., Crespo, A.: Wind turbine wake aerodynamics. Prog. Aerosp. Sci., vol. 39: (2003) pp.467-510.

[2] Sørensen, J.N.: Aerodynamic aspects of wind energy conversion. Annu. Rev. Fluid Mech., vol 43: (2011) pp. 427-48.

[3] van Kuik, G.A.M., et al.: Long-term research challenges in wind energy - a research agenda by the European Academy of Wind Energy. Wind Energ. Sci., vol. 1: (2016) pp. 1-39.

[4] Stevens, R.J.A.M., Meneveau, C.: Flow Structure and Turbulence in Wind Farms. Annu. Rev. Fluid Mech., vol. 49: (2017) pp: 311-39.

[5] Cal, R.B., et al.: Experimental study of the horizontally averaged flow structure in a model windturbine array boundary layer. Journal of renewable and sustainable energy, vol. 2, no. 013106: (2010) pp.1-25.

[6] Zhang, W., et al.: Near-wake flow structure downwind of a wind turbine in a turbulent boundary layer. Exp Fluids, vol. 52: (2012) pp. 1219-1235. 
[7] Krogstad, P.-A., Lund, J.A.: An experimental and numerical study of the performance of a model turbine. Wind Energ., vol. 15: 2012, pp. 443-457.

[8] Okulov V.L., et al.: A regular Strouhal number for large-scale instability in the far wake of a rotor. J. Fluid Mech., vol. 747: (2014) pp. 369-380.

[9] Calaf, M., Meneveau, C., Meyers, J.: Large eddy simulations of fully developed wind-turbine array boundary layers, Phys. Fluids., vol. 22, no. 015110: (2010) pp.1-16.

[10] Wu, Y., Porté-Agel, F.: Large-Eddy Simulation of Wind-Turbine Wakes: Evaluation of Turbine Parametrisations. Boundary-Layer Meteorol, vol. 138: (2011) pp. 345-366.

[11] Churchfield, M.J., et al.: A numerical study of the effects of atmospheric and wake turbulence on wind turbine dynamics. Journal of Turbulence. vol. 13, no. 14: (2012) pp. 1-32.

[12] Martínez-Tossas, L.A., et al.: Large eddy simulations of the flow past wind turbines: actuator line and disk modeling. Wind Energ., vol.18, no. 6: (2014) pp.1047-1060.

[13] Munters, M., et al.: Turbulent Inflow Precursor Method with Time-Varying Direction for LargeEddy Simulations and Applications to Wind Farms. Boundary-Layer Meteorol. vol. 159: (2016) pp.305-328.

[14] Mehta, D., et al.: Large eddy simulation of wind farm aerodynamics: a review. J. Wind Eng. Ind. Aerodyn. vol. 133: (2014) pp.1-17.

[15] Kolmogorov, A.N.: Proc.: Math. Phys. Sci., vol. 32: (1941) pp. 16-18.

[16] Pope, S.B.: Turbulent Flows. Cambridge University Press: (2000). p.749.

[17] Kolmogorov, A.N.: A refinement of previous hypotheses concerning the local structure of turbulence in a viscous incompressible fluid at High Reynolds number. J. Fluid Mech., vol. 13: (1962) pp. 82-85.

[18] Vassilicos, J.C.: Intermittency in Turbulent Flows. Cambridge University Press: (2001).

[19] Frisch, U., Sulem, P.-L., Nelkin, M.: A simple dynamic model of intermittent fully developed turbulence. J. Fluid Mech., vol. 87: (1978) pp. 719-736.

[20] Vindel, J.M., Yage, C., Redondo, J. M.: Structure function analysis and intermittency in the atmospheric boundary layer. Nonlinear Processes Geophys., vol. 15: (2008) pp.915-929.

[21] Stresing, R., Peinke, J., Seoud, R. E., Vassilicos, J. C.: Defining a New Class of Turbulent Flows. PHYSICAL REVIEW LETTERS, vol. 104, no. 194501: (2010) pp.1-4.

[22] Ali, N., et al.: Structure functions, scaling exponents and intermittency in the wake of a wind turbine array. Journal of renewable and sustainable energy, vol. 8: (2016) p. 013304.

[23] Sagaut, P.: Large eddy simulation for incompressible flows: an introduction. Springer, Berlin: (2002) p. 426.

[24] Meneveau, C., Lund, T.S., Cabot, W.H.: A Lagrangian dynamic subgrid-scale model of turbulence. J. Fluid. Mech., vol. 319: (1996) pp.353-385.

[25] Sørensen, J.N., Shen, W.Z.: Numerical Modeling of Wind Turbine Wakes, Journal of Fluids Engineering, vol. 124: (2002) pp. 393-399.

[26] Weller, H.G., et al.: A tensorial approach to computational continuum mechanics using object oriented techniques, Computers in Physics, vol.12, no. 6: (1998) pp. 620-631.

[27] Tijera, M., Cano, J. L., Cano, D., Bolster, D., and Redondo, J. M. (2008). Filtered deterministic waves and analysis of the fractal dimension of the components of the wind velocity. Nuovo Cimento C. Geophysics and Space Physics, 31, 653-667.

[28] Leyton, C., J. M. Redondo, P.L. Gonzalez-Nieto and A.M. Tarquis. (2016) Fractal Behaviour of Human Fluxes, Waves and vortices in complex media, Ishlinski IPM RAS. Moscow. P. 2, 230345.

[29] Ciuchi F., L Sorriso-Valvo, A Mazzulla, J M Redondo: Fractal aggregates evolution of methyl red in liquid crystal.The European Physical Journal E 07/2009; 29(2):139-47.

[30] Tarquis, A. M., Platonov, A., Matulka, A., Grau, J., Sekula, E., Diez, M., \& Redondo, J. M. (2014). Application of multifractal analysis to the study of SAR features and oil spills on the ocean surface. Nonlinear Processes in Geophysics, 21(2), 439-450.

[31] Castilla R., Redondo J.M., Gamez P.J. and Babiano A. (2007), Non Linear Processes in Geophysics, 14, 139. 